Pacific Journal of Mathematic 


\section{THE USE OF FORMS IN VARIATIONAL CALCULATIONS}

\section{Louis Auslander}

Introduction. The purpose of this paper is to present a method of calculating the first and second variation which is suitable for spaces which have a Euclidean connection. I then use this method to calculate the first and second variations along a geodesic in a Finsler space in terms of differential invariants of the Finsler metric. In the special case of Riemannian geometry, this calculation has been carried out by Schoenberg in [4].

Indications as to how this calculation should be made are originally due to E. Cartan [1]. I wish to thank Prof. S. S. Chern for the privilege of seeing his calculations on this matter for Riemann spaces.

1. Algebraic Preliminaries. Let $I=[0,1]$ and $0 \leq \xi_{1}, \xi_{2} \leq 1$. Let $M^{n}$ be an $n$-dimensional $C^{\infty}$ manifold. Assume we have a one parameter family of mappings of $I$ into $M^{n}$ which we will denote by $f\left(\xi_{1}, \xi_{2}\right)$, where $\xi_{2}$ is taken as the parameter along $I$ and $\xi_{1}$ parametrizes the family of mappings. Then we may define a mapping $\eta: I \times I \rightarrow M^{n}$ by the equation

$$
\eta\left(\xi_{1}, \xi_{2}\right)=f\left(\xi_{1}, \xi_{2}\right) .
$$

We require that $\eta$ shall also be a $C^{\infty}$ mapping.

Let $\eta_{*}$ denote the mapping induced by $\eta$ on the tangent space to $I \times I$ into the tangent space to $M^{n}$. Let $\eta^{*}$ denote the dual mapping induced on the cotangent spaces. Then we define two vector fields $X_{1}$ and $X_{2}$ over $\eta(I \times I)$ by

$$
X_{2}=\eta_{*}\left(\partial / \partial \xi_{2}\right) \text { and } X_{1}=\eta_{*}\left(\partial / \partial \xi_{1}\right) .
$$

Then if $w$ is any form in $M^{n}$ we may write

$$
\eta^{*}(w)=w_{\delta} d \xi_{1}+w_{d} d \xi_{2},
$$

where $w_{\delta}$ and $w_{c}$ are defined by the equation.

Lemma 1.1. If $\langle X, w\rangle$ denotes the value that $X$ takes on the covector $w$ at each point, then

$$
w_{\delta}=\left\langle X_{1}, w\right\rangle
$$

and

$$
w_{i}=\left\langle X_{2}, w\right\rangle .
$$

Received February 12, 1954 and in revised form June 2, 1954. 
Proof. $\quad w_{\delta}=\left\langle\partial / \partial \xi_{1}, \eta^{*}(w)\right\rangle=\left\langle\eta^{*}\left(\partial / \partial \xi_{1}\right), w\right\rangle=\left\langle X_{1}, w\right\rangle$.

The proof is analogous for $w_{d}$.

Let $\Omega$ be any two form and let $X_{1}$ and $X_{2}$ be any two vector fields. It is well known that $\Lambda^{2}(V)$ and $\Lambda^{2}\left(V^{*}\right)$ are dually paired. Let this pairing be denoted by

$$
\left\langle X_{1} \wedge X_{2}, \Omega\right\rangle \text {. }
$$

Then if $\Omega$ can be decomposed as $w_{1} \wedge w_{2}$, where $w_{1}$ and $w_{2}$ are one forms, we have that the pairing may be defined by the following expression:

$$
\left\langle X_{1} \wedge X_{2}, w_{1} \wedge w_{2}\right\rangle=\left\langle X_{1}, w_{1}\right\rangle\left\langle X_{2}, w_{2}\right\rangle-\left\langle X_{1}, w_{2}\right\rangle\left\langle X_{2}, w_{1}\right\rangle .
$$

Theorem 1.1.

$$
\left\langle X_{1} \wedge X_{2}, w_{1} \wedge w_{2}\right\rangle=w_{1 \delta} w_{2 d}-w_{1 d} w_{2 \delta} .
$$

The proof of this theorem is straightforward.

We define the symbols $\delta w_{d}$ and $d w_{\delta}$ by the following equations:

$$
\begin{aligned}
& \delta w_{d}=\partial / \partial \xi_{1}\left\langle X_{2}, w\right\rangle, \\
& d w_{\delta}=\partial / \partial \xi_{2}\left\langle X_{1}, w\right\rangle .
\end{aligned}
$$

If $f$ is any function of $\xi_{1}$ and $\xi_{2}$, we define

$$
d^{r} \delta^{s} f=\frac{\partial^{t} f}{\partial \xi_{2}^{r} \partial \xi_{1}^{s}},
$$

where $t=r+s$. Define $\delta^{r} d^{s} f$ similarly.

$$
\text { THEOREM 1.2. }\left\langle X_{1} \wedge X_{2}, d w\right\rangle=\delta w_{d}-d w_{\delta} .
$$

Proof. Now, in terms of a local coordinate system $\left(x_{1}, \cdots, x_{n}\right)$,

$$
\left\langle X_{1} \wedge X_{2}, d w\right\rangle=\sum\left[\frac{\partial}{\partial \xi_{1}}\left(a_{i} \frac{\partial x_{i}}{\partial \xi_{2}}\right)-\frac{\partial}{\partial \xi_{2}}\left(a_{i} \frac{\partial x_{i}}{\partial \xi_{1}}\right)\right]
$$

since

$$
\sum a_{i} \frac{\partial^{2} x_{i}}{\partial \xi_{1} \partial \xi_{2}}=\sum a_{i} \frac{\partial^{2} x_{i}}{\partial \xi_{2} \partial \xi_{1}}
$$

This and the definition of $\delta w_{d}$ and $d w_{\delta}$ prove the theorem.

2. The First Variation. Consider the integral

$$
I=\int_{a}^{b} F\left(q_{1}, \cdots, q_{n} ; q_{1}^{\prime}, \cdots, q_{n}^{\prime} ; t\right) d t
$$

in a space $M$ of $2 n+1$ dimensions. Then in the cotangent space to the manifold $M$ define the form $w$ by the equation 


$$
w=\sum \frac{\partial F}{\partial q_{i}^{\prime}} d q-\left(\sum q_{i}^{\prime} \frac{\partial F}{\partial q_{i}^{\prime}}-F\right) d t
$$

Now let $C$ be a curve in $M^{2 n+1}$ expressed by the equations

$$
q_{i}=q_{i}\left(\xi_{2}\right), \quad q_{i}^{\prime}=q_{i}^{\prime}\left(\xi_{2}\right), \quad t=(b-a) \xi_{2}+a .
$$

Assume further that $d q_{i} / d \xi_{2}=q_{i}^{\prime}$ for all values of $\xi_{2}$. Let $X_{2}$ be the image of $\partial / \partial \xi_{2}$ under the mapping described above. Then

$$
X_{2}=\sum q_{i}^{\prime} \frac{\partial}{\partial q_{i}}+\sum \frac{\partial q_{i}}{\partial \xi_{2}} \frac{\partial}{\partial q_{i}^{\prime}}+(b-a) \frac{\partial}{\partial t},
$$

and

$$
w_{d} d \xi_{2}=F\left(q, q^{\prime}, t\right) \underset{(b-a)}{d t} .
$$

Hence

$$
I=\int_{0}^{1} w_{d} d \xi_{\xi_{2}}=\int_{a}^{\prime} F\left(q_{1}(t), \cdots, q_{n}(t) ; q_{1}^{\prime}(t), \cdots, q_{n}^{\prime}(t) ; t\right) d t .
$$

Now consider a one parameter family of curves $f\left(\xi_{1}, \xi_{2}\right)$ each with the property described above. For each curve in the family we get a vector field which we will denote by $X_{2}\left(\xi_{1}\right)$. We may consider the variational problem for this family of curves. The crucial fact is that the requirement that $f\left(\xi_{1}, \xi_{2}\right)$ is a mapping of a fixed interval for each fixed value of $\xi_{1}$ enables us to treat the problem of variable end point without the necessity of differentiating limits of integration. We consider

$$
I\left(\xi_{1}\right)=\int_{0}^{1}\left\langle X_{2}\left(\xi_{1}\right), w\right\rangle d \xi_{2}
$$

and

$$
\delta I=\frac{\partial I\left(\xi_{1}\right)}{\partial \xi_{1}}=\int_{0}^{1} \partial w_{d} d \xi_{2}
$$

If we add and subtract $d w_{\delta}$ under the integral sign we get

$$
\begin{aligned}
\delta I & =\left[w_{\delta}\right]_{0}^{1}+\int_{0}^{1}\left(\delta w_{d}-d w_{\delta}\right) d \xi_{2} \\
& =\left[w_{\delta}\right]_{0}^{1}+\int_{0}^{1} w^{\prime}(\delta, d) d \xi_{2},
\end{aligned}
$$

where

$$
w^{\prime}(\delta, d)=\left\langle X_{1} \wedge X_{2}, d w\right\rangle
$$


and

$$
w^{\prime}(d, \delta)=\left\langle X_{2} \wedge X_{1}, d w\right\rangle \text {. }
$$

It may be noted that $w^{\prime}(\delta, d)=-w^{\prime}(d, \delta)$. The term $\left[w_{\delta}\right]_{0}^{1}$ is called the transversality term.

Theorem 2.1. Assume $\left[w_{8}\right]_{0}^{1}=0$. Then a necessary and sufficient condition for $\delta I=0$ for all variations is that $d w=0$ along $C$.

Proof. The condition is clearly sufficient. An equivalent form of the hypothesis is that

$$
\int_{0}^{1}\left\langle X_{1} \wedge X_{2}, d w\right\rangle d \xi_{2}=0
$$

for all vector fields $X_{1}$ along $C$. Assume $d w$ does not equal zero along $C$. Then there exists an $X_{1}$ such that $\left\langle X_{1} \wedge X_{2}, d w\right\rangle>0$ for some open interval $a<\xi_{2}<b$. Then we may choose a new vector field $X_{1}$ such that:

$$
\begin{aligned}
& \bar{X}_{1}=X_{1} \quad \text { for } \quad a<\xi_{2}<b \\
& \bar{X}_{1}=0 \quad \text { for } \quad 0 \leq \xi_{2} \leq a-\varepsilon \quad \text { or } \quad b+\varepsilon \leq \xi_{2} \leq 1 \text {, }
\end{aligned}
$$

where $\varepsilon$ may be chosen arbitrarily small. Then

$$
\int_{0}^{1}\left\langle\bar{X}_{1} \wedge X_{2}, d w\right\rangle d \xi_{2}=\int_{a}^{b}\left\langle X_{1} \wedge X_{2}, d w\right\rangle d \xi_{2}+\varepsilon^{\prime},
$$

where $\varepsilon^{\prime}$ depends on $\varepsilon$ and $\lim _{\varepsilon \rightarrow 0} \varepsilon^{\prime}=0$. Hence we may choose $\varepsilon$ in such a way that

$$
\left.\int_{0}^{1}\left\langle\bar{X}_{1} \wedge X_{2}, d w\right\rangle d \xi_{2}\right\rangle 0
$$

This contradiction proves the theorem.

Remark: This is essentially the usual argument for the derivation of Euler's equation.

3. Application to Finsler Geometry. If we assume that our integral is of the Finsler type then we may proceed to calculate the second variation. For treating this special case we assume that the reader has a familiarity with Euclidean connections and we will use the Euclidean connection for a Finsler space as calculated by E. Cartan in [2] and Chern [3].

Let $M$ be an $n$-dimensional differentiable manifold and let $G$ be the principal bundle over $M$ with fiber and group the $n$-dimensional orthogonal groups, $O_{(x)}$. Then in $G$, we have forms $w_{i}, w_{i j}$, where $w_{i j}+$ $w_{j i}=0$ and $i, j=1, \cdots, n$. The equations of structure are 


$$
\begin{aligned}
& d w_{i}=w_{j} \wedge w_{j i}+\gamma_{j i \alpha} w_{j} \wedge w_{a n} \\
& d w_{i j}=w_{i k} \wedge w_{k j}+\Omega_{i j},
\end{aligned}
$$

where $\alpha=1, \cdots, n-1$. (Henceforth we will assume that Greek indices run from 1 to $n-1$ and Latin indices run from 1 to $n$.) The $\gamma_{i j a}$ are symmetric in all indices and zero if any index is $n$. Also

$$
\Omega_{i j}=\frac{1}{2} \sum_{\alpha, \beta} Q_{i j \alpha \beta} w_{\alpha n} \wedge w_{\beta n}+\sum_{l, \alpha} P_{i j l x} w_{l} \wedge w_{\alpha n}+\frac{1}{2} \sum_{l, k} R_{i j l k} w_{l} \wedge w_{k} .
$$

Let $C$ be any path in $M^{n}$. Choose any path in $G$ with the property that if $e_{1}, \cdots, e_{n}$ represents a righthanded frame, that is, an element of $O_{(n)}$, then $e_{n}$ is in the tangent direction to $C$. Then arc length along a path $C$ is

$$
I=\int_{0}^{1}\left(w_{n}\right)_{a} d \xi_{2} .
$$

This follows from equation (2.4) and the definition of $w_{n}$ (see [3]).

Now $X_{2}=e_{n}$ and $X_{1}=\sum k_{i} e_{i}$. Therefore $\left(w_{n}\right)_{\delta}=\left\langle X_{1}, w_{n}\right\rangle=k_{n}$. Hence if $X_{1}$ is perpendicular to the curve $C$, then the transversality term is zero. From equation (3.1), we have

$$
d w_{n}=\sum w_{\alpha} \wedge w_{\alpha_{n}} .
$$

Hence

where

$$
\delta I=\left[\delta\left(w_{n}\right)\right]_{0}^{1}+\int_{0}^{1} \sum\left\{\left(w_{\alpha}\right)_{\delta}\left(w_{\alpha n}\right)_{d}-\left(w_{\alpha}\right)_{d}\left(w_{\alpha n}\right)_{\delta}\right\} d \xi_{2},
$$

where

$$
\left(w_{a}\right)_{d}=\left\langle w_{\alpha}, e_{n}\right\rangle=0 .
$$

It is clear from the last equation that the symbols $\delta$ and $d$ and our indices make the notation awkward. Hence a $w_{d}$ will be written as $w$ and a $w_{\delta}$ will be written as $\phi$. In this notation equation (3.4) becomes

$$
I=\left[\phi_{n}\right]_{0}^{1}+\int_{0}^{1} \sum \phi_{\alpha} w_{a n} d \xi_{2},
$$

since $w_{\alpha}=0$ along the path $C$.

From Theorem 2.1 we have the following theorem.

Theonem 3.1. The differential equations of a geodesic in Finsler geometry are

$$
w_{\alpha}=0, \quad w_{a n}=0, \quad \alpha=1, \cdots, n-1 .
$$

We will now compute the second variation along a geodesic. We have 


$$
\delta I=\int_{0}^{1} \delta w_{n} d \xi_{2}
$$

and $\delta^{2} I$ is the second variation. Hence we have to compute $\delta^{2}\left(w_{n}\right)$ along a geodesic. Now

$$
\delta^{2}\left(w_{n}\right)=\delta d\left(\phi_{n}\right)+\phi_{\alpha} \delta\left(w_{a n}\right)
$$

since $w_{\alpha n}=0$ along the geodesic. We have

$$
\delta\left(w_{a n}\right)-d\left(\phi_{a n}\right)=\left\langle X_{1} \wedge X_{2}, d w_{\alpha_{n}}\right\rangle .
$$

From equation (3.2) we obtain

$$
\left\langle X_{1} \wedge X_{2}, d w_{\alpha n}\right\rangle=\left\langle X_{1} \wedge X_{2}, w_{\alpha \beta} \wedge w_{\beta n}\right\rangle+\left\langle X_{1} \wedge X_{2}, \Omega_{\alpha n}\right\rangle .
$$

By Theorem 1.1 and since $C$ is a geodesic, we have

$$
\delta w_{\alpha n}=d \phi_{\alpha n}-w_{\alpha \beta} \phi_{\beta n}+\left\langle\Omega_{\alpha n}, X_{1} \wedge X_{2}\right\rangle .
$$

Now by equation (3.2) and the facts that

$$
R_{i j k l}=-R_{j i k l}, \quad R_{i j, k l}=R_{k l, i j}
$$

we have

$$
\left\langle X_{1} \wedge X_{n}, \Omega_{\alpha n}\right\rangle=\sum P_{n \alpha n \beta} w_{n} \phi_{\beta n}+\sum R_{n \alpha n \beta} \phi_{\beta} w_{n} .
$$

Therefore, from equations (3.6), (3.8) and (3.9), we obtain

$$
\delta^{2}\left(w_{n}\right)=\delta d \phi_{n}+\sum \phi_{\alpha}\left[d \phi_{\alpha n}-\phi_{\beta n} w_{\alpha \beta}+P_{n \alpha n \beta} w_{n} \phi_{\beta n}+R_{n \alpha n \beta} \phi_{\beta} w_{n}\right] .
$$

Now,

$$
\delta d \phi_{n}=d \delta \phi_{n} \quad \text { and } \quad d\left(\phi_{\alpha} \phi_{\alpha n}\right)=\phi_{\alpha n}\left(d \phi_{\alpha}\right)+\phi_{\alpha}\left(d \phi_{\alpha n}\right) .
$$

Hence

$$
\begin{aligned}
\delta^{2}\left(w_{n}\right)= & d\left[\delta \phi_{n}+\phi_{\alpha} \phi_{\alpha n}\right]-\phi_{\alpha n} d \phi_{\alpha} \\
& +\left[-\phi_{\alpha} \phi_{\beta n} w_{\alpha \beta}+P_{n \alpha n \beta} \phi_{\alpha} \phi_{\beta n}+R_{n \alpha n \beta} \phi_{\alpha} \phi_{\beta}\right] w_{n} .
\end{aligned}
$$

But from equation (3.1) we have

$$
d \phi_{\alpha}=\delta w_{\alpha}+w_{j} \phi_{j \alpha}-\phi_{j} w_{j \alpha}
$$

since

$$
\gamma_{j \alpha \beta}\left[\phi_{j} w_{\beta n}-w_{j} \phi_{\beta n}\right]=0
$$

along the geodesic. Also $\delta w_{\alpha}=0$ along the geodesic, since $w_{\alpha} \geq 0$ and equals zero along the geodesic and hence $w_{\alpha}$ must attain a minimum along a geodesic. 


\section{Hence}

$$
\delta^{2} w_{n}=d\left[\delta \phi_{n}+\sum \phi_{\alpha} \phi_{\alpha n}\right]+\sum\left(\phi_{\alpha n} \phi_{\alpha n}+P_{n \alpha n \beta} \phi_{\alpha} \phi_{\beta n}+R_{n a n \beta} \phi_{\alpha} \phi_{\beta}\right) w_{n} .
$$

Hence the integral form of the second variation becomes

$$
\delta^{2} I=\left[\delta \phi_{n}+\sum \phi_{\alpha} \alpha_{\alpha n}\right]_{0}^{1}+\int_{0}^{1} \sum\left(\phi_{\alpha n} \phi_{\alpha n}+P_{n \alpha n \beta} \phi_{\alpha} \phi_{\beta n}+R_{n \alpha n \beta} \phi_{\alpha} \phi_{\beta}\right) w_{n} d \xi_{2} .
$$

For Riemannian geometry we have $P_{i j k l}=0$ and $\sum \phi_{\alpha} \phi_{\alpha n}$ represents the second fundamental form of the geodesic surface perpendicular to the geodesic at the point.

\section{REFERENCES}

1. E. Cartan, Leçons sur les invarients integraux, Paris, Hermann, (1922).

2. _- Les Espaces de Finsler, Actualités Scientifiques et Industrielles, no. 79, Paris, Hermann, (1934).

3. S. S. Chern, On the Euclidean connections in a Finsler space, Proc. Nat. Acad. Sci., 29 (1943), 33-37.

4. I. J. Schoenberg, Some applications of the calculus of variations to Riemannian geometry, Ann. of Math., 33 (1932), 485-495.

YALE UNIVERSITY 

.././. ./FrontMatter/paper .pdf 


\section{Pacific Journal of Mathematics}

Nesmith Cornett Ankeny and Theodore Joseph Rivlin, On a theorem of S.

Bernstei........................................ 849

Louis Auslander, The use of forms in variational calculation .......... 853

Paul Civin, Abstract Riemann sum . .......................... 861

Paul Civin, Some ergodic theorems involving two operator ............ 869

Eckford Cohen, The number of solutions of certain cubic congruence .... . 877

Richard M. Cohn, Specializations over difference field .............. 887

Jean Dieudonné, Pseudo-discriminant and Dickson invarian . . ......... 907

Ky Fan, A comparison theorem for eigenvalues of normal matrice ........ 911

Richard P. Gosselin, On the convergence behaviour of trigonometric interpolating polynomial ........................... 915

Peter K. Henrici, On generating functions of the Jacobi polynomial . . . . . . . 923

Meyer Jerison, An algebra associated with a compact grou ............ 933

Wilhelm Magnus, Infinite determinants associated with Hill's equatio . . . . . 941

G. Power and D. L. Scott-Hutton, The slow steady motion of liquid past a semi-elliptical bos................................. 953

Lyle E. Pursell, An algebraic characterization of fixed ideals in certain function ring .................................... 963

C. T. Rajagopal, Additional note on some Tauberian theorems of O. Szás . . 971 Louis Baker Rall, Error bounds for iterative solutions of Fredholm integral

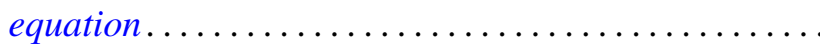

Shigeo Sasaki and Kentaro Yano, Pseudo-analytic vectors on

pseudo-Kählerian manifold ......................

Eugene Schenkman, On the tower theorem for finite group

P. Stein and John E. L. Peck, On the numerical solution of Poisson's equation over a rectangl ........................

Morgan Ward, The mappings of the positive integers into themselves which preserve divisio .

Seth Warner, Weak locally multiplicatively-convex algebra 1025

Louis Weisner, Group-theoretic origin of certain generating function .... 